\title{
Fluidisation des particules granuleuses et des flocons dans l'eau
}

\author{
The fluidization of granulated particles \\ and flocs in water
}

\author{
PAR IGOR TESARIK \\ INGÉNIEUR a L'INSTTTtT D'HYDRODYNAMIQUE DE L'ACADÉMIE DES SCIENCES DE TCHÉCOSLOVAQUTE
}

\begin{abstract}
I'étude d'un problème posé à l'Institut d'Hydrodynamique de l'Académie des Sciences de Tchécoslovaquie pour des besoins industriels a permis à l'auteur de dégager quelques idées générales sur la fluidisation des particules en suspension dans l'eall. Dans le trauail présenté, ont été incluses les expériences faites avec des conches fluidisées composées, soit de particules identiques (spheres), soit de particules dont la forme et les dimensions sont absolument irrégulières (flocons).

Pendant la fluidisation dans l'eau, on a trouvé une relation linéaire entre la bitesse moyenne réelle et la porosité, le poids apparent de la couche étant approximativement égal à la trainée.

Le processus de la fluidisation est considéré comme un éconlement autour des sphères individuelles dont est composé le lit. A l'aide de la méthode de l'analyse dimensionnelle, l'auteur est parvenul à une équation assez simple, sans dimension, entre les relations des coefficients de trainée et des nombres de Reynolds. Du point de vue hydraulique, le procédé nouveau du traitement de l'eau brute, connu sous le nom de "décantation dans le lit de boue», fait partie du phénomène de la fluidisation. La suspension floculée est attirée à la surface des flocons du lit de boue de la mème façon qu'elle est retenue par adsorption par les grains de sable all sein d'un filtre rapide. Les observations ont démontré que, pour une mème quantité d'eau brute et pour une même dose de réactifs, les concentrations de la boue s'affaiblissent avec l'augmentation de la vitesse de traversée du lit. Le régime de l'écoulement a été reconnu turbulent aut sein du lit de boue et laminaire au-dessus de sa surface. L'énergie des fuctuutions dans le lit cause le bris des flocons et leur mélange, ce qui produit la diminution du degré de turbulence de bas en haut. Les expériences exécutées dans l'installation d'essais ont servi a la construction d'un nouveau type de décanteur à lit de boule.
\end{abstract}

\begin{abstract}
As a result of the Hydrodynamic Institute of the Czechoslovak Academy of Sciences having been asked to study a problem arising in industry, the author was able to define certain general concepts concerning the fluidization of particles in saspension in water. The work described in this paper includes experiments concerned with fluidized beds consisting either of identical particles (spherical) or of completely irregular particles (flocs).
\end{abstract}

For fluidization in water, an approximately linear relationship was found between the mean real velocity and porosity; apparent weight being approximately equal to drag.

The process of fluidization is considered to be flow around the individual spheres making up the bed. Using dimensional analysis the author has obtained a comparatively simple equation involving dimensionless quantities and connecting the drag coefficients and Reynolds numbers.

As far as hydraulics is concerned, the new water treatment known as sludge blanket treatment is a fluidization phenomenon. The focular supension is attracted to the surface of the flocs forming the sludge blanket in the sane way as it is held inside a rapid filter bl sand adsorption. It was seen that, for a given quantity of untreated water and for a given quantity of reagent, sludge concentration diminished as flow velocities through the blanket increased. Flow in the sludge blanket was seen to be turbulent flow above the blanket uas laminar. The energy resulting from fluctuations in the blanket causes the flocs to break up and mixes them with a resultant decrease in turbulence in the upper parts of the sludge blanket.

The results of experiments conducted with the test installation were used in designing a new type of sludge blanket clarifier. 


\section{NOTATIONS EMPLOYEES}

$\mathrm{D}_{p}$ : Diametre de la particule.

D : Diamètre de la tuyauterie cylindrique.

L : Hauteur du lit.

F : Section de la luyauterie.

$\mathrm{U}$ : Vitesse apparente moyenne du fluide.

$\mathrm{U}_{\epsilon}$ : Vitesse réelle moyenne du fluide.

$\Delta P$ : Perte de charge dans le lit.

$Q$ : Débit de l'eau.

$g$ : Accélération de la pesanteur.

\author{
$3_{.}$: Densité de la particule. \\ : Densité du milieu fluide. \\ :. : Viscosité dynamique du fluide. \\ $n:$ Nombre des sphères. \\ s : Porosité du lit. \\ $\mathrm{C}_{x^{\prime}}$ : Coefficient de traînée. \\ Q : Nombre de Reynolds. \\ o : Exposant. \\ c, C: Constantes.
}

\section{INTRODUCTION}

Depuis quelque lemps on a commencé à utiliser les propriétés des lits lluidisés pour dirférentes applications techniques. Le terme « flüdisation » s'applique à des conches mobiles constituées de particules solides ou de flocons, faisant partie de suspensions traversées par un courant ascensionnel du fluide.

Dans un lit fluidisé, la poussée équilibre le poids des particules dans le fluide; les particules constituant le lit ont une mobilité extrème, landis que la surface supérieure du lit est nettement séparée du fluide.

Due à la mobilité du lit fluidisé, la répartition des propriétés du fluide et des particules présentes à l'intérieur de l'installation est presque uniforme; le contact du solide et de l'agent fluidisant au sein du lit devient très intime. Ces propriétés du lit ont une grande importance dans les applications industrielles : contact optimal de la surface du catalyseur avec le fluide dans les réactions hétérogènes, augmentation considérable des coefficients de transfert de chaleur dans les réacteurs, facilité de manutention du solide fluidisé pour le transport en conduite, etc.

O'n se sert aussi des lits fluidisés pour le lavage des filtres rapides; à présent, on utilise les lits fluidisés de flocons pour la décantation des impuretés présentes dans l'eau brute (décanteurs à «lit de boue »).

En ce qui concerne la concentration des particules, une couche fluidisée rappelle le charriage des matériaux sur le fond d'une rivière.

Depuis la dernière guerre mondiale, on a monté beaucoup d'installations pilotes et industrielles dans différentes applications techniques.
Malgré les nombreuses études publiées sur ce thème, la théorie hydrodynamique des couches fluidisces n'est pas encore développée.

Dans l'étude présentée, l'auteur a essayé de généraliser les résultats de ses essais par la méthode d'analyse dimensionnelle.

D'aprés une analyse cinématique et dynamique, on distingue trois processus portant le nom de fluidisation :

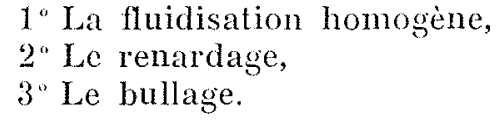

1. Pendant la fluidisation homogène, les parlicules sont séparées l'une de l'autre de façon presque uniforme. Avec une vitesse moyenne constante de l'écoulement, il n'y a que des variations modérées de la hauteur de la couche autour d'une valeur moyenne. Dans le lit, l'intensité du mouvement turbulent devient beaucoup plus grande que dans un fluide sans particules, pour une même vitesse moyenne.

$2^{\circ}$ S'il y a une surface très étendue dans une unité du volume du lit, l'écoulement n'est pas divisé uniformément dans toute l'installation. Evidemment, c'est la coalescence qui fait unir les particules, pendant que la plus grande partie du fluide s'écoule dans les canaux.

$3^{\circ}$ Pendant le bullage, le fluide passe à l'intérieur de l'installation sous forme de bulles, qui font monter devant elles un nuage de particules, comme un piston; le piston ayant atteint une hauteur critique, se détache et les particules tombent.

Ce phénomène a été observé seulement sous 
fluidisation par le gaz, ce qui conduit à supposer qu'il est causé par la compressibilité du gaz.

Dans le travail présenté ici, on a compris les expériences avec des couches fluidisées de sphères identiques et de flocons dans l'eau. Ces deux phénomènes représentent les cas le plus simple ef le plus difficile de la fluidisation particuliere.

Le diamètre des sphères examinées a toujours été plus grand que $0,1 \mathrm{~mm}$; c'est pourquoi on a négligé les forces électrostatiqes et de Van der Waals, lesquelles jouent un grand rôle dans le mouvement des particules colloïdales.

\section{FLUIDISATION DES LITS DE SPHÊRES IDENTIQUES}

La fluidisation des lits de particules granuleuses est caractérisée par un mouvement, dont la turbulence est intense. Les particules visualisent les tourbillons dans l'écoulement, ce qui donne une impression de grouillement absolument désordonné. Le dépouillement des observations effectuées par une «photocamera » nous a montré gue les fluctuations de la vitesse instantanée à chaque point du lit suivent la loi de la probabilité. Les vitesses et les porosités moyennes à chaque point de la couche fluidisée restent approximativement constantes. En ce qui concerne la vitesse relative entre le fluide et les particules, la fluidisation ne diffère pas de la sédimentation libre d'un nuage de particules ayant une même concentration. La répartition uniforme des vitesses moyennes est causée par la présence de particules dont la surface par unité de volume excède la surface motillée de linstallation.

Un accroissement de la vitesse moyenne de l'écoulement à l'intérieur du lit fluidisé fait augmenter sa hauteur et sa porosité. Nous avons tracé les courbes caractérisant la relation de la porosité $\varepsilon$ avec la vitesse moyenne réelle:

$$
\mathrm{U}_{\epsilon}=\mathrm{U} / \varepsilon
$$

pour les sortes et les nombres les plus différents des sphères (fig. 1).

Pendant la fluidisation homogène, la courbe caractérisant la relation des vitesses réelles moyennes en fonction de la porosité, est pratiquement une droite. Selon les vitesses d'écoulement, le maximum de la porosité de la couche

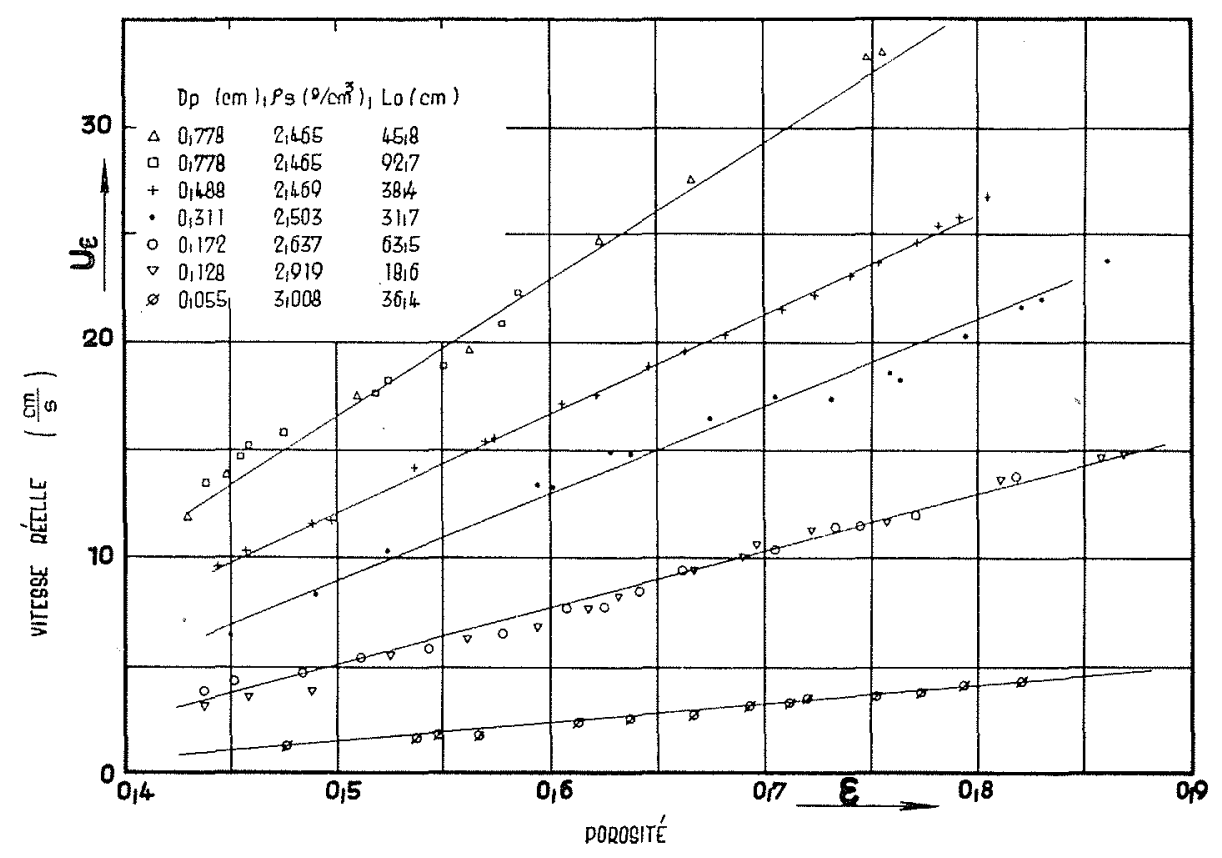

FIG. 1

Porosité des lits tluidisés de sphères identiques en fonetion de la vitesse ascensionnelle. 


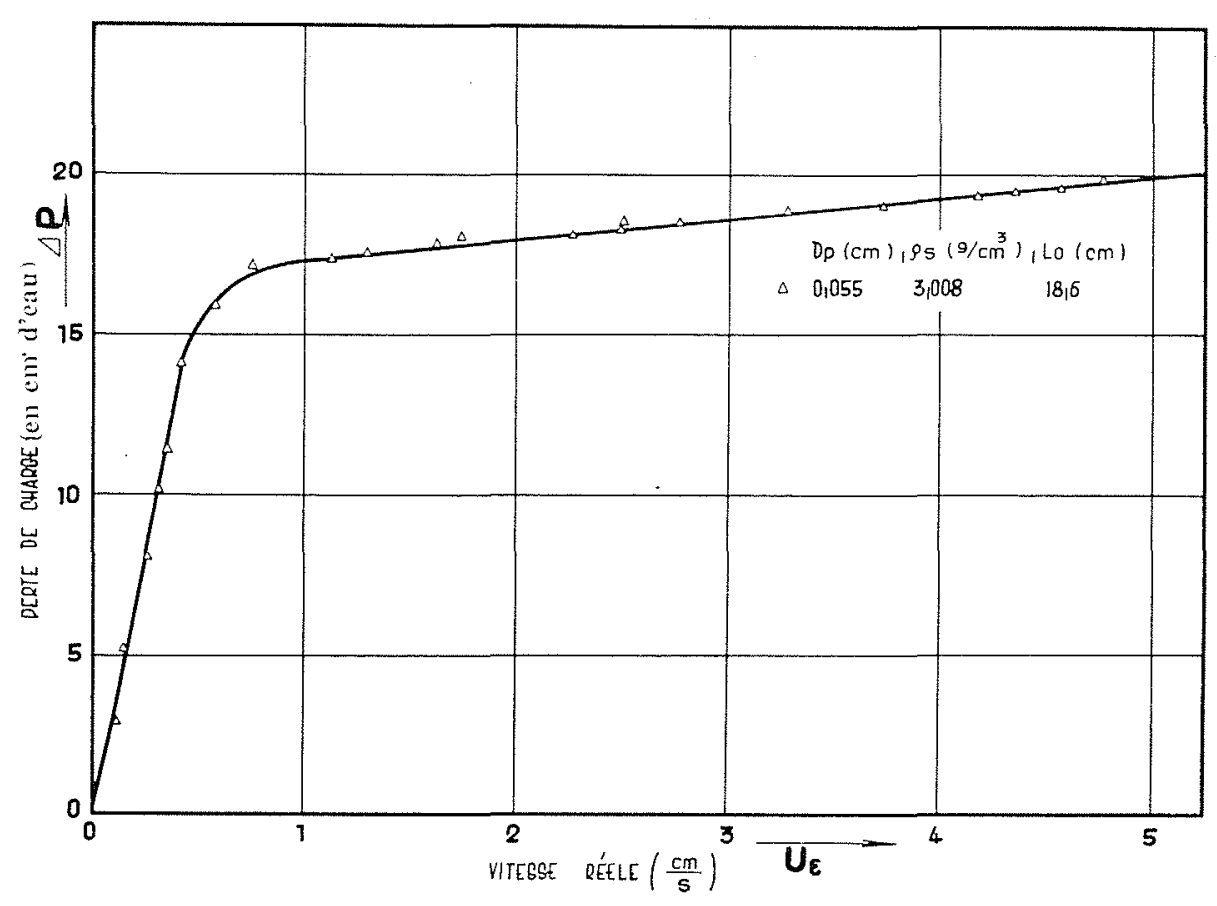

Fici. 2

Perle de charge pendant l'écoulement dans lo lit fixe et fluidisé (sphères, $D_{n^{\prime}}=0,55 \mathrm{~mm}$ ).

fluidisée doit être de 0,80-0,90. Si l'on fait écouler le liquide avec une vitesse excessive, la porosilé du lit augmente; les particules quittent le fond, le lit s'étend et tout le phénomène se transforme en transport de particules en suspension.

La figure 2 montre la perte de charge pendant l'écoulement dans un lit fixe et fluidisé en fonction de la vitesse réelle. En augmentant la vitesse de l'écoulement dans un lit fixe à partir de zéro, la traînée (représentée yar la perte de charge) grandit jusqu'au moment où elle atteint le poids apparent des particules. A ce moment, le lit commence à se fluidiser.

Par conséquent, il y a dans une couche fluidisée un équilibre entre le poids apparent et la traînée causée par les forces de pression et de frottement hydraulique.

La perte de charge pendant la fluidisation homogène augmente très modérément en raison de la dissipation d'énergie produite par les fluctuations turbulentes de l'eau et des particules. Supposons que, d'après la chute libre des grains isolés dans un fluide, le poids apparent soit proportionnel au maitre couple et au carré de la vitesse réelle moyenne d'écoulement, suivant l'équation :

$$
g\left(\rho_{s}-\rho\right)\left(\pi \mathrm{D}_{p}{ }^{3} / 6\right)=\mathrm{C}_{x} \cdot\left(\pi \cdot \mathrm{D}_{p}{ }^{2} \cdot \rho \cdot \mathrm{U}_{\epsilon}{ }^{2}\right) / 8
$$

Néanmoins, la présence d'autres particules dans le fluide et les violentes fluctuations turbulentes influencent la valeur $d u$ coefficient de trainée et la vitesse réelle moyenne.

\section{Calcul d'analyse dimensionnelle}

Les expériences montrent que le fluide ayant des propriétés physiques constantes traverse le lit fluidisé dont la configuration et les dimensions sont déterminées, sous l'action des forces extérieures dans un certain champ de vitesses moyennes.

Grâce à celte propriété, on peut résoudre le problème de la fluidisation au moins par le calcul d'analyse dimensionnelle.

Supposons que le processus de la fluidisation puisse être considéré comme un écoulement autour des sphères individuelles dont le lit est composé. Choisissons comme longueur caractéristique de l'écoulement le diamètre de la sphère $\mathrm{D}_{p}$. Utilisons la vitesse réelle moyenne comme vitesse caractéristique. Comme force extérieure, agissant à l'intérieur du lit, on ne peut choisir que la traînée; cette force est représentée approximativement par la perte de charge totale $\Delta P$. Les quantilés caractérisant les propriétés physiques du fluide sont représentées par la viscosité $\mu$ et par la densité p, Pour que nous puissions comparer les résultats expérimentaux avec des nombres différents de particules composant la couche fluidisée, nous rapporterons la perte de charge totale $\Delta \mathrm{P}$ à une seule sphère. Le phénomène examiné peut ètre exprimé par l'équation :

$$
\Delta \mathrm{P} / n=c . \mathrm{F}\left(\mathrm{U}_{\epsilon}, \mu, \rho, \mathrm{D}_{p}\right) .
$$


La pesanteur et la densité des grains ne sont pas comptés parmi les dimensions caractéristiques; leur effet est déjà compris dans la vitesse réelle moyenne.

On suppose une relation exponentielle entre les quantités caractéristiques :

$$
\Delta \mathrm{P} / n=:=c \cdot\left(\mathrm{U}_{\epsilon}\right)^{\alpha} \cdot(\mu)^{\beta} \cdot(\mathrm{o})^{\gamma} \cdot\left(\mathrm{D}_{p}\right)^{\beta}
$$

Il est facile de voir que :

$$
\alpha=\hat{\delta}, \beta=2-\delta, \gamma=\delta-1 \text {. }
$$

Un calcul d'analyse dimensionnelle conduit au groupement suivant des variables:

où :

$$
\mathrm{C}_{x}=(8 c / \pi) \cdot \vec{R}^{\delta--2}
$$

$$
\mathscr{R}=\hat{q} \cdot \mathrm{U}_{\epsilon}, \mathrm{D}_{p} / \mu=\text { nombre de Reynolds, }
$$$$
\mathrm{C}_{x}=8 \Delta \mathrm{P} / n \cdot \pi \cdot \mathrm{D}_{p}{ }^{2} \cdot \mathrm{q} \cdot \mathrm{U}_{\mathrm{e}}
$$

$=$ coefficient de trainée d'une sphère.

La figure 3 montre, en coordonnées logarithmiques, la variation du coefficient de traînée en fonction du nombre de Reynolds, pour les essais pratiqués avec des lits fluidisés de sphères identiques dans notre tuyauterie expérimentale. Le tableau $A$ donne des renseignements sur la nature des couches examinées. Les points d'essais sont bien groupés autour des courbes de même allure. On voit que l'exposant o.-2 de l'équation (3) demeure constant pour chaque catégorie de sphères.

La famille de courbes dans lesquelles s'insèrent les points expérimentaux de $\mathrm{C}_{i}$ et $\mathcal{R}$ quant aux propriétés physiques différentes peut être explicitée comme suit :

$$
\log \mathrm{C}_{x}=\log \mathrm{C}+(\delta-2) \log \mathcal{R},
$$

où $\delta-2=-1,95$ pour toutes les catégories de sphères,

$$
\mathrm{C}=8 \mathrm{c} / \pi=\text { constante. }
$$

En passant aux exponentielles, on obtient la formule :

$$
\mathrm{C}_{w}=\mathrm{C} \cdot \boldsymbol{R}^{-1,95}
$$

A l'aide du calcul d'analyse dimensionnelle, nous sommes parvenus à une équation sans dimension assez simple, entre le coefficient de traìnée et le nombre de Reynolds. Il faut encore analyser la valeur de C. Il est évident (fig. 3) que la valeur de $C$, étant constante pour chaque vitesse de fluidisation pour une catégorie de particules, n'est pas la même pour une autre sorte de sphères.

La figure 3 donne aussi la valeur du coefficient

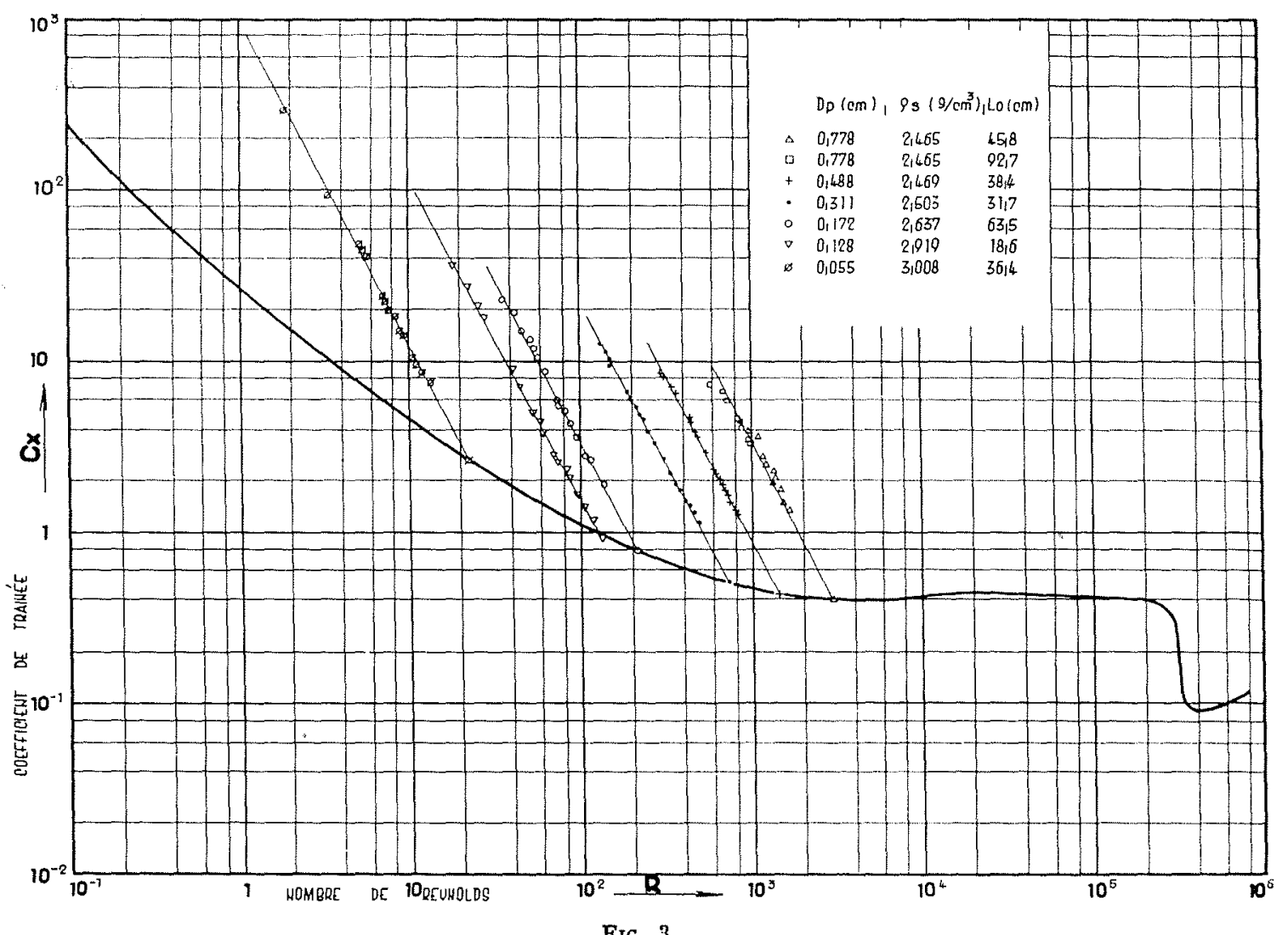

Variation du coefficient de traînéce en fonction du nombre de Reynolds, pour les jits fluidisés de sphères identiques. 
TABLEAU A .

\begin{tabular}{|c|c|c|c|c|c|c|c|c|c|c|c|}
\hline \multicolumn{6}{|c|}{ Caractéristiques d'une sphère } & \multicolumn{3}{|c|}{ Caractéristiques du lit } & \multicolumn{3}{|c|}{ Caractéristiques du fluide } \\
\hline Matériel & Densité & Diamètre & $\begin{array}{l}\text { Vitesse } \\
\text { de chute }\end{array}$ & $\begin{array}{c}\text { Nombre } \\
\text { de Reynolds }\end{array}$ & $\begin{array}{l}\text { Coefficient } \\
\text { de trainée }\end{array}$ & $\begin{array}{l}\text { Hauteur } \\
\text { initiale }\end{array}$ & $\begin{array}{l}\text { Porosité } \\
\text { initiale }\end{array}$ & $\begin{array}{l}\text { Diametre de } \\
\text { la tuyauterie }\end{array}$ & Liquide & Densité & Viscosité \\
\hline & $P_{s}$ & $\mathrm{D}_{p}$ & $\mathbf{U}_{0}$ & $\mathfrak{R}_{0}$ & $\mathrm{C} x_{0}$ & $\mathrm{~L}_{\mathrm{c}}$ & $\varepsilon_{0}$ & $\mathrm{D}$ & & p & $\mu$ \\
\hline & $g / \mathrm{cm}^{3}$ & $\mathrm{~cm}$ & $\mathrm{~cm} / \mathrm{s}$ & - & - & $\mathrm{cm}$ & - & $\mathrm{cm}$ & & $g / \mathrm{cm}^{3}$ & $\mathbf{c P}$ \\
\hline Verre & 2,503 & 0,311 & 36,2 & 751,0 & 0,468 & 31,7 & 0,427 & 20 & l'eau & 1,001 & 1,496 \\
\hline Verre & 2,469 & 0,488 & 46,4 & 211,0 & 0,437 & 38,4 & 0,430 & 20 & l'eau & 1,001 & 1,539 \\
\hline Verre & 2,465 & 0,778 & 68,2 & 315,8 & 0,372 & 45,8 & 0,437 & 20 & l'eau & 1,000 & 1,683 \\
\hline Verre & 2,465 & 0,778 & 68,2 & 315,8 & 0,372 & 69,4 & 0,442 & 20 & l'eau & 1,000 & 1,683 \\
\hline Verre & 2,465 & 0,778 & 68,2 & 315,8 & 0,372 & 92,7 & 0,444 & 20 & l'eau & 1,000 & 1,683 \\
\hline Verre & 2,919 & 0,128 & 18,6 & 134,2 & 0,934 & 18,6 & 0,412 & 20 & l'eau & 1,001 & 1,771 \\
\hline Verre & 2,919 & 0,128 & 18,6 & 134,2 & 0,934 & 27,3 & 0,400 & 20 & l'eau & 1,001 & 1,771 \\
\hline Verre & 2,919 & 0,128 & 18,6 & 134,2 & 0,934 & 37,8 & 0,423 & 20 & l'eau & 1,001 & 1,771 \\
\hline Verre & 3,020 & 0,116 & 17,5 & 111,9 & 1,000 & 16,3 & 0,419 & 20 & l'eau & 1,003 & 1,803 \\
\hline Verre & 3,008 & 0,055 & 7,78 & 23,3 & 2,455 & 18,6 & 0,432 & 20 & l'eau & 1,003 & 1.833 \\
\hline Verre & 3,008 & 0,055 & 7,78 & 23,3 & 2,455 & 36,4 & 0,417 & 20 & l'eau & 1,003 & 1,833 \\
\hline $\mathrm{CaCO}_{3}$ & 2,637 & 0,172 & 22,9 & 215,0 & 0,700 & 42,6 & 0,433 & 20 & l'eau & 1,003 & 1,833 \\
\hline $\mathrm{CaCO}_{3}$ & 2,637 & 0,172 & 22,9 & 215,0 & 0,700 & 63,5 & 0,429 & 20 & l'eau & 1,003 & 1,833 \\
\hline
\end{tabular}




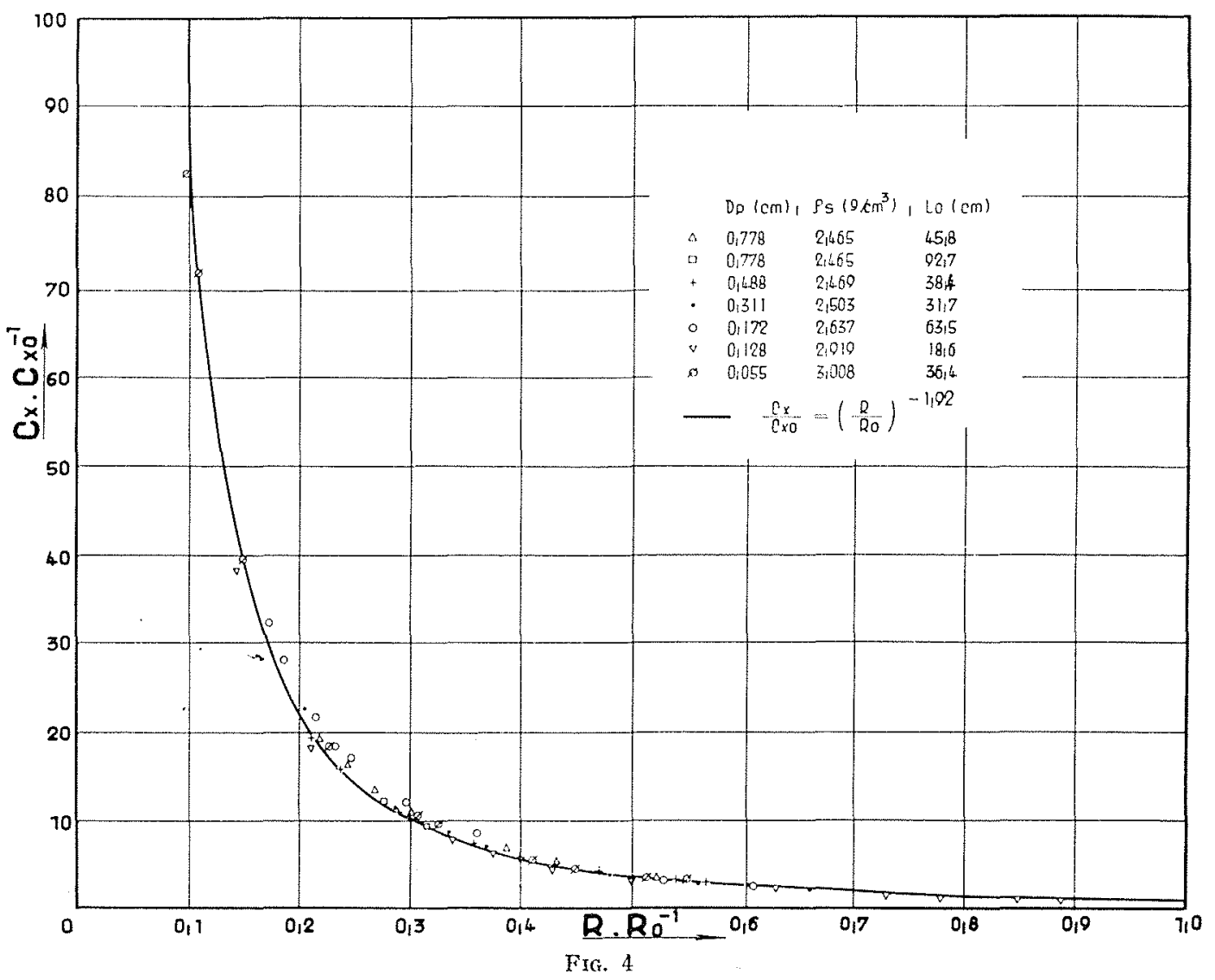

Comparaison entre les points d'essais et l'équation générale de la fludisation des sphères identiques.

de traînée en fonction du nombre de Reynolds pour deux séries d'essais effectués avec des sphères identiques, dont le nombre de Reynolds est différent.

Les points expérimentaux des deux essais se groupent autour d'une courbe. Nous en déduisons que la constante $C$ pour une catégorie de sphères ne dépend pas de leur nombre.

Il résulte de la figure 3 que la constante $C$ reste identique pour chaque porosité de la suspension, c'est-à-dire qu'elle est valable aussi dans le cas où l'écoulement commence à entraîner les particules en suspension, dont la concentration est faible.

Nons avons déterminé la vitesse de chute (tableau A) d'une sphère isolée pour tous les matériaux examinés et constaté que la constante $\mathrm{C}$, correspondant aux propriétés physiques des particules examinées et du fluide, reste invariable même dans le cas où une sphère isolée tombe en chute libre (fig. 3 , points placés sur la courbe qui représente la variation du coefficient de trâ̂née en fonction du nombre de Reynolds).

La particularité de ce phénomène nous servira pour sa solution générale. La solution gé- nérale qui nous intéresse est une relation dans laquelle il n'y a plus la constante C.

Suivant notre considération précédente, on obtiendra l'élimination de la constante $\mathrm{C}$ en divisant chaque valeur du coefficient de traînée $\mathrm{C}_{x}$ et du nombre de Reynolds $\mathcal{R}$ par un coefficient étalon $C_{x_{0}}$ et un nombre étalon $\mathcal{A}_{0}$ qui correspondent à la vitesse de chute libre d'une sphère isolée de même sorte dans le fluide.

Une série d'essais avec un lit fluidisé sera représentée par les équations :

$$
\begin{aligned}
& { }^{1} \mathrm{C}_{m i} / \mathrm{C}_{i: 0}=\mathrm{C}_{n l} \mathrm{~F}\left({ }^{1} \mathfrak{R}\right) / \mathrm{C}_{a} \mathrm{~F}\left(\mathcal{R}_{0}\right) \\
& { }^{2} \mathrm{C}_{i j} / \mathrm{C}_{a, l}=\mathrm{C}_{n} \mathrm{~F}\left({ }^{2} \mathfrak{R}\right) / \mathrm{C}_{a l} \mathrm{~F}\left(\mathscr{R}_{0}\right) \\
& { }^{j} \mathrm{C}_{n} / \mathrm{C}_{x_{0}}=-\mathrm{C}_{a} \mathrm{~F}\left({ }^{j} \mathfrak{\alpha}\right) / \mathrm{C}_{a} \mathrm{~F}\left(\mathfrak{R}_{0}\right)
\end{aligned}
$$

où les indices $1,2, \ldots j$, désignent les essais sous différentes vitesses. Cette considération peut être étendue à toutes les catégories de sphères (caractérisées par les constantes $\mathrm{C}_{a}, \mathrm{C}_{l}, \ldots \mathrm{C}_{k}$ ).

Il est évident que la fonction $\mathrm{F}$ reste la même que celle mentionnée ci-dessus, c'est-à-dire exponentielle, avec l'exposant égal à --1,95.

Par conséquent, le phénomène de la fluidisa- 
tion des sphères identiques peut être exprimé par l'équation générale :

$$
\mathrm{C}_{x} / \mathrm{C}_{x o}=\left(\mathfrak{R} / \mathcal{R}_{0}\right)^{-1,95}
$$

La figure 4 montre que les points d'essais s'ordonnent très bien autour de la courbe représentant l'équation (6). Le point terminal de la courbe se rapporte au cas de chute libre d'une sphère isolée; il en résulte qu'en ce point, les rapports $\mathrm{C}_{\mathrm{x}} / \mathrm{C}_{w_{i}}, \mathfrak{R} / \mathcal{R}_{0}$ doivent être égaux à 1 .

\section{Description de l'installation d'essais et de méthodes de mesure}

Nos expériences ont été effectuées dans un tube vertical en plexiglas (A) dont la hauteur est de $2880 \mathrm{~mm}$ et le diamètre de $200 \mathrm{~mm}$. La figure 5 est un schéma de l'installation.

Une pompe refoule un débit maximum de $10 \mathrm{l} / \mathrm{s}$ d'eau dans un bac à niveau constant (B). Le débit constant s'écoule par un conduit d'un diamètre de $90 \mathrm{~mm}$ dans le bas de la tuyauterie transparente, dans laquelle est placée une toile régulière en cuivre (C), réalisant une distribution aussi uniforme que possible des vites-

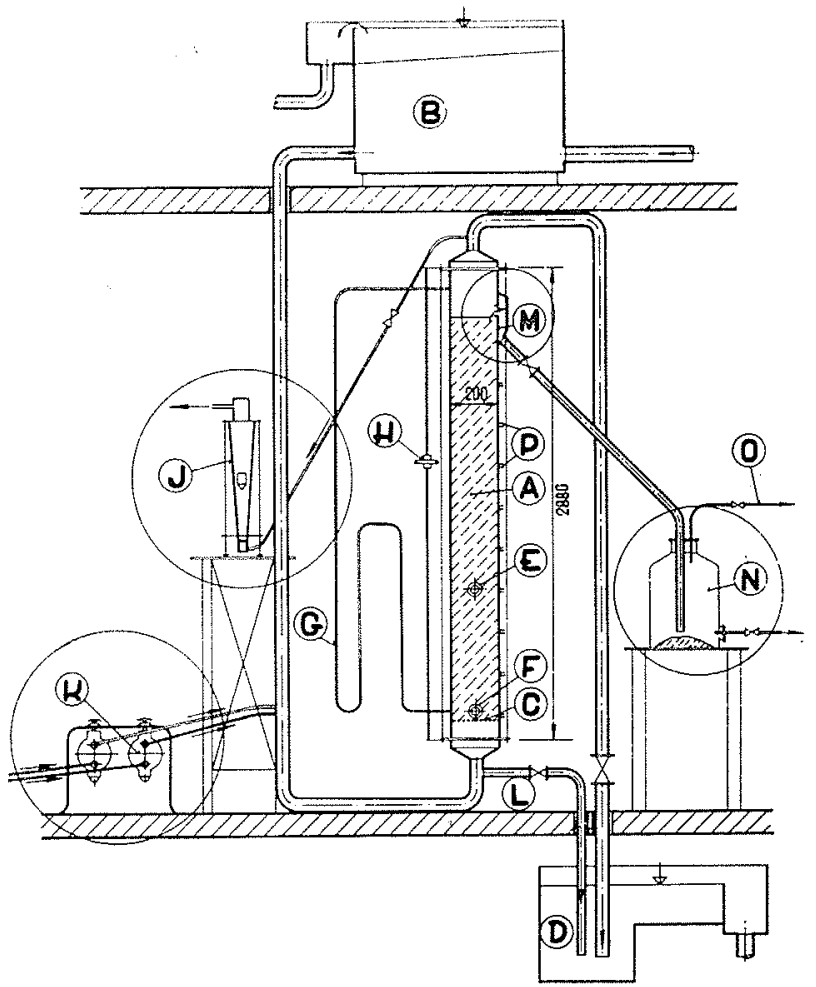

Fig. 5

Schéma de l'installation d'essais. Les appareils $J, K, M, N$, étaient en fonctionnement seulement: pendant la fluidisation des flocons.

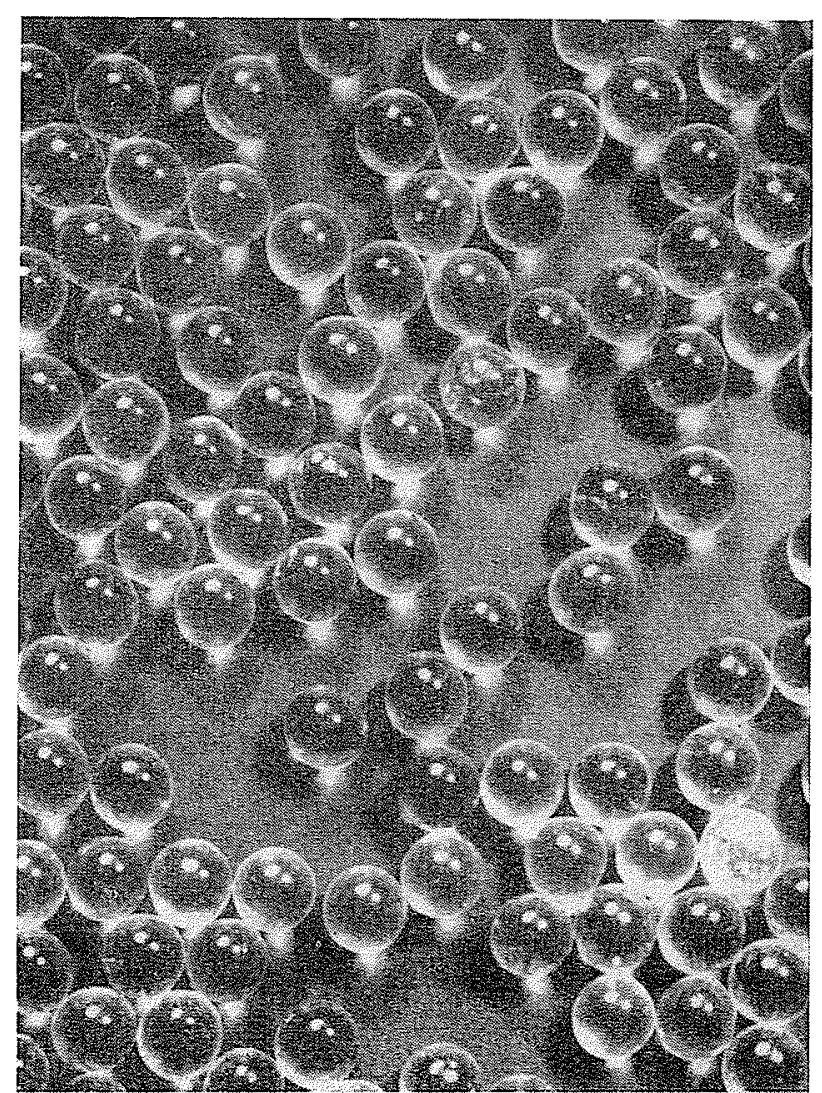

FIG. 6

Unitormilé des sphères identiques $\left(D_{n}=0,311 \mathrm{~cm}\right)$.

ses moyennes; les fils de la toile sont à une distance de $0,25 \mathrm{~mm}$ les uns des autres. Le tube expérimental en plexiglas est relié au conduit par un divergent conique dans le bas, et par un convergent dans le haut. Le débit de l'eau est mesuré dans un déversoir triangulaire à $90^{\circ}$ (D) placé à l'aval de la tuyauterie expérimentale. Les matériaux examinés sont introduits dans le tube par l'ouverture (E), qui se trouve à une distance d'environ $100 \mathrm{~cm}$ de la toile. L'essai terminé, les sphères sont retirées du tube expérimental par une ouverture placée audessus de la toile. L'installation peut être entièrement vidée par le conduit $(\mathrm{F})$.

Pour connaître la perte de charge dans un lit fluidisé, on détermine la différence des pressions entre deux points : l'un, situé tout près de la partie inférieure de la toile, el l'autre, dans la partie supérieure du tube expérimental, par un manomètre de Prandtl à eau $(G)$.

Pendant les essais, nous avons utilisé des sphères ayant une densité et un diamètre constants. Les matériaux ont été triés grâce à des tamis à maille carrée; la figure 6 montre l'uniformité des sphères. La moyenne arithmétique de 50 sphères représente la dimension de chaque lit.

Les densités absolues moyennes ont été mesu- 
rées au picnomètre et le diamètre des sphères, all micromètre.

Le nombre des sphères a été facilement calculé d'après le poids, le diamètre et la densité des sphères.

La hauteur exacte du lit a été mesurée par un appareil spécial $(\mathrm{H})$ composé d'une échelle verticale, divisée en degrés d'un millimètre, et munie d'un cathétometre. Dans chaque position de la lunette de celui-ci, son axe optique passe par l'axe vertical de la tuyauterie d'essais. Pendant un essai, l'axe optique de la lunette est fixé de manière à pouvoir observer le niveau du lit, la hauteur étant lue sur l'échelle.

La porosité du lit composé de sphères identiques est déterminée d'après la formule:

$$
\varepsilon=1-\left[\left(2 n \mathrm{D}_{\eta}^{3}\right) /\left(3 \mathrm{LD}^{2}\right)\right]
$$

La viscosité dynamique du fluide est mesurée au viscosimètre de Höppler.

\section{FLUIDISATION DES LITS DE FLOCONS}

C'est grâce aux progrès réalisés dans le domaine de l'épuration des èaux (par la mise en cuvre des coagulants, le dosage automatique, le progrès dans les dispositifs de décantation et dans les procédés de correction chimique de l'eau traitée, etc.), qu'on a pu sans crainte avoir recours aux eaux superficielles pour l'alimentation en. eau des centres urbains.

Depuis peu, on a commencé à utiliser le procédé de la décantation des flocons dans un lit fluidisé (lit de boue) au lieu de la décantation statique.

L'eau superficielle est ordinairement fortement chargée de matières en suspension et de matières colloïdales. La quantité de ces matières très fines est indiquée par la turbidité et la couleur de l'eau. Les particules fines présentes dans l'eau brute possèdent ordinairement une charge électronégative. Les forces électrostatiques de répulsion qui règnent entre les particules collö̈dales ont tendance à maintenir la distance qui les sépare. Etant très faibles, les forces électrostatiques n'agissent qu'à une distance insignifiante. Pour qu'on puisse éliminer les particules produisant la turbidité et la couleur de l'eau, il est donc nécessaire de neutraliser leurs charges électriques.

Grâce aux progrès décisifs accomplis dans la science des colloïdes, on sait que les hydrates de fer ou d'alumine forment, pour une valeur définie du $\mathrm{pH}$, des édifices micellaires garnis d'ions, dont le signe est opposé à celui des micelles de l'eau brute.

Le mélange des coagulants avec l'eau brute produit donc le déchargement des deux collö̈des, ce qui a pour conséquence la formation de « microfloes », c'est-à-dire de particules à peine perceptibles, qui, selon leurs dimensions, font partie des suspensions. On peut supposer que les forces électrostatiques des microflocs sont déjà négligeables, mais que leur surface spécifique devient énorme.

L'expérience a montré que les fluctuations modérées de la iurbulence favorisent la floculalion, en approchant les microflocs les uns des autres à une distance minime. Ce sont probablement les forces de Van der Waals qui occasionnent l'union des microflocs, en produisant des particules de l'ordre de quelques millimètres.

Le but de la floculation est de produire une cau débarrassée des particules colloïdales et des suspensions fines. Les particules floculées sont retenues à la surface des flocons fluidisés par les mêmes forces qu'au sein d'un filtre rapide. A la condition d'un bon fonctionnement du lit Iluidisé, l'eau traitée ne contient que $5 \%$ des impuretés initiales. L'efficacité et le fonctionnement des décanteurs à lit de boue sont alors beaucoup plus avantageux que celle des bassins de sédimentation.

Contrairement au cas ci-dessus cité (fluidisation de sphères identiques), la taille des flocons du lit de boue est tout à fait irrégulière et leurs dimensions s'étendent de $0,1 \mathrm{~mm}$ jusqu'à $10 \mathrm{~mm}$. Une autre difficulté importante résulte de la fragilité des flocons : par conséquent, l'intensité des fluctuations turbulentes doit être faible; sinon, les fluctuations briseraient le floc constitué pendant la floculation. En même temps, la vitesse de l'écoulement traversant le lit fluidisé de flocons doit être petite, parce que la densité des flocons n'excède que de très peu celle de l'eau. Avant de faire une installation pour le traitement des eaux, il faut bien choisir le procédé d'épuration. Ce sont généralement les méthodes de laboratoire qui servent de base à la conception des installations pour le traitement des eaux. Cependant, ces méthodes ne permettent pas de savoir comment l'eau se comportera pen- 
dant le procédé de traitement continu et quelle sera son efficacité.

A mi-chemin, entre la méthode de laboratoire et la pratique industrielle, se situent les études sur modèle réduit. Le modèle réduit présente l'avantage de permettre l'observation de l'eau traitée en continu.

Pour que nous puissions observer l'influence de différents facteurs du traitement sur la décantation des flocons dans le lit fluidisé, nous avons apporté les modifications suivantes à notre tuyauterie d'essais (fig. j) : on a refoulé l'eau brute dans le bac (B) à niveau constant. Le tuyau conduisant l'eau au déversoir triangulaire (D) a été fermé par une vanne. Le débit de l'cau traitée traversant le lit de boue a élé amené par un tuyau souple à faible section dans un rolametre où on l'a mesuré (J). Nous avons amené à la base de notre tube expérimental un débit constant de $100 \mathrm{~cm}^{3} / \mathrm{s}$ d'cau brute. Par un appareil automatique de dosage (K) fondé sur le principe d'une pompe-membrane, nous avons introduit, sous forme de solution, la dose de réactifs (sulfate d'alumine additionné de silice activée, ou chlorure ferrique) produisant la floculation optimale de l'eau brute. La dose optimale a été déterminée dans un floculateur de laboratoire. Pour qu'or puisse changer la vitesse d'écoulement traversant le lit de boue, on a laissé échapper une partie du débit constant de l'eau floculée en actionnant la vanne (L). En même temps, on a diminué le débit s'écoulant dans le rotametre. Au commencement de nos essais, on a rempli le tube expérimental jusqu'à la toile avec de l'eau brule, puis, par l'ouverture $(E)$, on a introduit la boue. L'ouverture (E) fermée, on a introduit un débit constant d'eau dans le tube expérimental, de facon à mettre la boue en suspension. En même temps, on a mis en fonctionnement l'appareil de dosage (K). Dû à l'affluence des flocons nouveaux, le lit augmente; la limite de séparation devient nette entre la couche de boue et le liquide clair. Pour qu'on puisse maintenir le lit à une hauteur constante, on préléve par le déversoir (M) une partie du débit constant qui est amené dans le bas du récipient $(\mathrm{N})$, dont le volume est de $15 \mathrm{l}$. La boue sédimentée est vidangée périodiquement, tandis qu'un quantum de $10 \%$ de l'eau traitée est évacué par le tube $(O)$, placé dans le haut du récipient $(\mathrm{N})$.

Tous les $200 \mathrm{~mm}$, le tủbe expérimental est muni d'ouvertures (P) fermées par des robinets. Par prélèvement d'échantillons, on détermine la variation de la concentration de boue le long du tube expérimental.

Le phénomène du lit de boue dépend d'un si grand nombre de parametres de l'eau brute (quantité des matières en suspension el colloïdales, pH, etc.), que sa complexiti est immense. Si l'on ne veut s'exposer à de très importantes erreurs dues à des interprétations injustifiées, il est donc nécessaire d'effectuer des essais continus et de procéder à des mesures extrêmement fréquentes.

La campagne de mesures a été menée pendant les mois de juillet, août el septembre 1957 et les observations ont été effectuées toutes les deux heures. Pendant une observation, on a mesuré : le débit et la turbidité de l'eau traversant le lit, le débit de la boue concentrée dans le bas du récipient, le débit et la turbidité de l'eau claire s'écoulant par le tube fixé dans le haut du récipient, la turbidité, le $\mathrm{pH}$ de l'eau brute, la floculation au-dessus du réseau et audessus du lit, la dose des réactifs.

Une attention toute particulière a été réservée aux variations de la concentration de boue aux différentes hauteurs du lit. Nous avons développé une méthode volumétrique pour la mesure de la concentration : à l'aide des tubes (P), on a prélevé des échantillons aux différentes hauteurs du lit. Le liquide boueux (floculat de sulfate d'alumine ou de chlorure ferrique) a été placé dans un tube de verre cylindrique de $100 \mathrm{~cm}^{3}$, gradué en volumes. On a mesuré la variation du rolume de boue sédimentée en fonction du temps. Le simple fait de prélever l'échantillon de boue de la tuyauterie d'essais dans le tube de verre a brisé les flocons. On a constaté que le volume de la boue du lit fluidisé, observé après deux heures de décantation dans la bouteille, ne changeait presque plus. Le rapport du volume occupé par la boue après la décantation de deux heures, au volume total de la bouteille $\left(100 \mathrm{~cm}^{3}\right)$ est appelé concentration apparente. Cependant, la concentration apparente ne détermine pas la concentration réelle de la boue du lit. Naturellement, la vitesse de décantation dans la bouteille dépend de la forme et des dimensions du récipient, la masse de boue se comprimant avec une vitesse qui est nécessaire à l'expulsion de l'eau interstitielle.

Nous avons aussi essayé d'appliquer une méthode couramment pratiquée: les échantillons, après une filtration et une dessiccation de deux heures (à la température de $105^{\circ} \mathrm{C}$ ), ont été pesés. Contrairement à la méthode précédente, nous avons constaté un manque de concordance dans les résultats obtenus par cette dernière méthode. Ce manque de concordance est dû à la présence irrégulière de particules granuleuses fines (dont la densité excède de beaucoup celle des flocons).

La méthode de mesure de la concentration apparente a permis d'analyser au moins 140 ćchantillons par jour et a fourni des résultats très satisfaisants. Nous avons utilisé cette méthode pour toutes les observations.

La vitesse moyenne de l'écoulement traver- 
sant le lit a été supposée « à vide » aussi, c'esta-dire, calculée d'après le rapport simple:

$$
\mathrm{U}=\mathrm{Q} / \mathrm{F} \text {. }
$$

La turbidité de l'eau a été mesurée par le turbidimètre précis de Pulfrich. Sa valeur a été exprimée en milligrammes de silice dans un litre d'eau ( $\left.\mathrm{mg} / \mathrm{l}, \mathrm{SiO}_{2}\right)$.

Les résultats de la floculation ont été transcrits avec les adjectifs : très bon, bon, médiocre, mal, insignifiant.

Nous avons effectué un grand nombre d'essais avec le lit de flocons fluidisé sous des vitesses différentes de l'eau, dont le contenu en matières colloïdales et les propriétés chimiques ont été divers. Pendant nos essais, on a aussi introduit des réactifs différents. Tous les essais ont été effectués sous une hauteur constante du lit de boue qui a été maintenue par l'aspiration de la boue excédentaire dans le récipient. Le débit traversant le lit étant changé, nous avons pu continuer nos observations au moment où le profil nouveau des concentrations s'est stabilisé. La stabilisation du lit sous un débit changé a duré quelques heures.

Les figures 7,8 présentent des relations typiques entre les vitesses à vide et les concentrations apparentes moyennes avec l'eau floculée au sulfate d'alumine additionnée de silice activée, d'une part, et avec le chlorure ferrique, d'autre part.

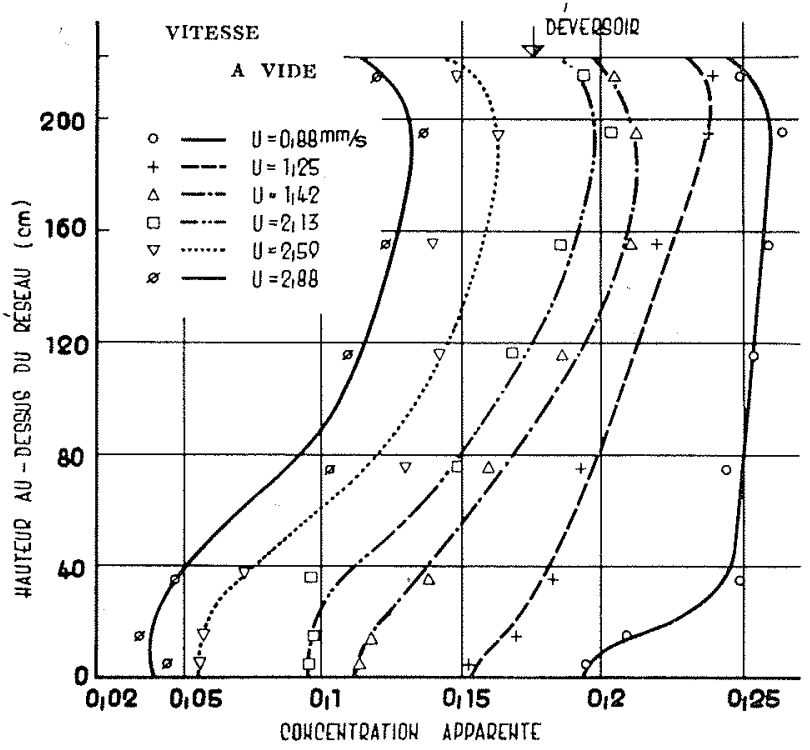

Fic. 7

Répartition des concentrations apparentes de la boue (floc de sulfate d'alumine additionné de silice alclivée) en fonction de la vitesse à vido

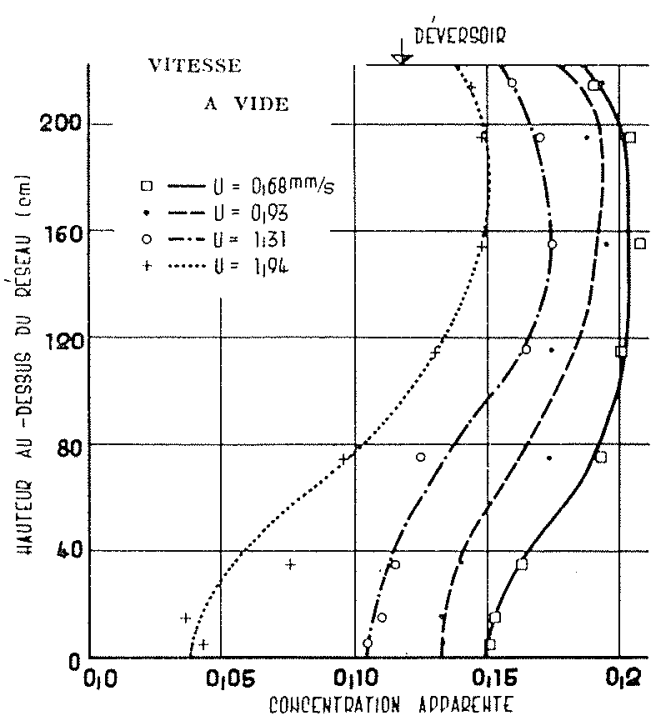

FIG. 8

Répartition des concentrations apparentes de la boue (floc de chlorure ferrique) en fonction de la vitesse à vide.

Sous le terme de concentration apparente moyenne, on comprend la moyenne arithmétique des sédiments, obtenus par la méthode décrite ci-dessus. Les tableaux B et $C$ donnent quelques renseignements sur les propriétés physiques et chimiques de l'eau brute et de l'eau traitée.

Les essais ont démontré qu'il n'y a pas de différences essentielles entre les processus hydrodynamiques dans les lits de flocons et dans les couches de matériaux granuleux.

Sous l'augmentation de la vitesse d'écoulement, la hauteur du lit de boue s'élève jusqu'à une certaine valeur d'expansion au-delà de laquelle le lit de boue commence à se briser. Après la rupture de la cohésion du lit, les morceaux de boue éparpillés sont entraînés par l'écoulement ascensionnel de l'eau. Les essais ont démontré que, sous une vitesse constante, avec des qualités invariables pour l'eau brute, avec une dose constante de réactifs et sous une hanteur identique du lit, les concentrations apparentes, à une hauteur définie du lit, ne varient presque pas. En ce qui concerne le profil des concentrations du lit de boue, on peut distinguer trois parties différentes: la partie inférieure (au-dessus de la toile), où les concentralions sont faibles, la partie centrale ayant une concentration élevée, et enfin la partie supérieure, dont l'épaisseur est relativement faible et la concentration décroissante. Il en résulte que les vitesses moyennes réelles dans les différentes hauteurs du lit ne sont pas identiques. La comparaison des figures 7,8 montre que, sous une mème vitesse à vide, la con- 
TABLEAU B

\begin{tabular}{|c|c|c|c|c|c|c|c|}
\hline \multirow[b]{2}{*}{$\begin{array}{c}\text { Température } \\
{ }^{\circ} \mathrm{C} \\
\end{array}$} & \multirow[b]{2}{*}{$\begin{array}{l}\text { Vitesse } \\
\text { at vide } \\
\mathrm{mm} / \mathrm{s}\end{array}$} & \multirow[b]{2}{*}{$\begin{array}{c}\text { Hauteur } \\
\text { du lit } \\
\text { mm }\end{array}$} & \multicolumn{2}{|c|}{$\begin{array}{c}\text { Turbidité : } \\
\text { Eau }\end{array}$} & \multirow[b]{2}{*}{$\begin{array}{c}\text { Dose de reactif } \\
\mathrm{Al}\left(\mathrm{SO}_{4}\right)_{3}+\mathrm{SiO}: \\
\mathrm{mg} / \mathrm{l}\end{array}$} & \multicolumn{2}{|c|}{ Floculation } \\
\hline & & & $\begin{array}{l}\text { brute } \\
\mathrm{mg} / \mathrm{l}\end{array}$ & $\begin{array}{l}\text { traitéc } \\
\mathrm{SiO}_{2}\end{array}$ & & $\begin{array}{l}\text { au-dessous } \\
\text { du lit }\end{array}$ & $\begin{array}{l}\text { au-dessus } \\
\text { du lit }\end{array}$ \\
\hline 17,6 & 0,88 & 2220 & 83,0 & 6,8 & $89 / 16$ & bon & 0 \\
\hline 15,3 & $1,2 \mathrm{i}$ & 2220 & 68,5 & 7,9 & $86 / 13$ & bon & 0 \\
\hline 18,2 & 1,25 & 2220 & 89,0 & 9,0 & $66 / 12$ & très bon & 0 \\
\hline 17,0 & 1,42 & 2220 & 82,5 & 18,5 & $83 / 14$ & bon & 0 \\
\hline 17,7 & 2,13 & 2220 & 120,0 & 34,0 & $84 / 11$ & bon & insignifiant \\
\hline 17,6 & 2,57 & 2220 & 110,0 & 40,0 & $80 / 14$ & bon & insignifiant \\
\hline 17,6 & 2,88 & 2220 & 96,0 & 51,3 & $\begin{array}{l}83 / 14 \\
\mathrm{FeCl}_{3}\end{array}$ & bon & insignifiant \\
\hline 14,3 & 0,68 & 2220 & 50,0 & 13,0 & 82 & bon & 0 \\
\hline 15,9 & 0,93 & 2220 & 58.0 & 14,9 & 85 & bon & 0 \\
\hline 15,8 & 1,31 & 2220 & $(65,5$ & 21,3 & 74 & bon & 0 \\
\hline 15,3 & 1,94 & 2220 & 57,3 & 28 & 79 & bon & 0 \\
\hline
\end{tabular}

centration dans le lit de floculat de sulfate d'alumine additionné de silice activée dépasse la concentration du lit de floculat de chlorure ferrique. En même temps, la turbidité de l'eau traitée par floculation 'de $\mathrm{Al}_{2}\left(\mathrm{SO}_{4}\right)_{3}$ avec $\mathrm{SiO}_{2}$ est plus faible que la turbidite de l'eau traitée par $\mathrm{FeCl}_{3}$, ce qui amène à supposer que l'effet de traitement par le lit de boue dépend de sa concentration volumétrique.

D’après les figures 7, 8, il est clair que les concentrations volumétriques s'affaiblissent avec l'augmentation de la vitesse traversant le lit; il en résulte que la turbidité de l'eau traitée devient plus grande (tableau B).

Le régime de l'écoulement a été déterminé par les observations avec une photocamera dans les hauteurs différentes du lit. Le dépouillement des films a montré que la présence de la toile provoque, au-dessus, des fluctuations turbulentes intenses du fluide. L'énergie des fluctuations cause le bris des flocons et leur mélange, ce qui produit, avec la dissipation de chaleur, la diminution du degré de turbulence. Cet effet est tres bien perceptible par la dimension des flocons, qui augmente avec la distance de la toile.

I'effet de l'affaiblissement du degré de turbulence au-dessus du lit est aussi accompagné par l'augmentation de la concentration de la boue (fig. 7, 8). La hauteur de la zone turbulente au-dessus de la toile dépend de la vitesse d'écoulement; elle peut atteindre une valeur maximum d'environ $50 \mathrm{~cm}$. Au-dessus de la zone de mélange, le degré de turbulence devient faible; la concentration volumétrique reste presque constante. On y a seulement observé des courants verticaux de flocons, dont quelquesuns descendent jusque dans la zone inférieure, turbulente. Le degré de turbulence au-dessous de la surface du lit est insignifiant. Lorsqu'il n'y a pas de particules quittant la surface du lit, il $y$ a une brusque différence entre les vitesses réelles dans le hant du lit et au-dessus.

TABLEAU C

\begin{tabular}{|c|c|c|c|c|c|c|c|c|c|c|c|}
\hline \multirow{2}{*}{$\begin{array}{l}\text { Vitesse } \\
\text { à vide } \\
\mathrm{mm} / \mathrm{s}\end{array}$} & \multirow{2}{*}{$\begin{array}{c}\text { Hauteur } \\
\text { du lit } \\
\\
\mathrm{mm}\end{array}$} & \multirow[t]{2}{*}{ Réactifs } & \multirow{2}{*}{$\begin{array}{l}\text { Dose de } \\
\text { réactifs } \\
\mathrm{mg} / \mathrm{l}\end{array}$} & \multicolumn{2}{|c|}{$\begin{array}{c}\text { pH } \\
\text { Eau }\end{array}$} & \multicolumn{2}{|c|}{$\begin{array}{l}\text { Acidité } \\
\text { Eau }\end{array}$} & \multicolumn{2}{|c|}{$\begin{array}{l}\text { Alcalinité } \\
\qquad \mathrm{Eau}\end{array}$} & \multicolumn{2}{|c|}{$\begin{array}{c}\text { Cousommation } \\
\text { en permanganate } \\
\text { Eau }\end{array}$} \\
\hline & & & & brute & traitée & $\begin{array}{l}\text { brute } \\
\text { m.é./1 }\end{array}$ & $\begin{array}{l}\text { traitée } \\
\text { m.é. } / 1\end{array}$ & $\begin{array}{l}\text { brute } \\
\text { m.é./l }\end{array}$ & $\begin{array}{l}\text { traitée } \\
\text { m.é./1 }\end{array}$ & $\begin{array}{l}\text { brute } \\
\mathrm{mg} / \mathrm{l}\end{array}$ & $\begin{array}{l}\text { traitée } \\
\qquad \mathrm{O}_{2}\end{array}$ \\
\hline $\begin{array}{l}2,13 \\
1,27\end{array}$ & $\begin{array}{l}2220 \\
2220\end{array}$ & $\begin{array}{c}\mathrm{Al}_{2}\left(\mathrm{SO}_{4}\right)_{3}+\mathrm{SiO}_{2} \\
\mathrm{FeCl}_{3}\end{array}$ & $\begin{array}{c}84 / 11 \\
74\end{array}$ & $\begin{array}{l}8,0 \\
7,5\end{array}$ & $\begin{array}{l}7,9 \\
6,8\end{array}$ & $\begin{array}{l}\text { alc. } \\
0,05\end{array}$ & 0,12 & $\begin{array}{l}1,2 \\
1,1\end{array}$ & $\begin{array}{l}0,8 \\
0,7\end{array}$ & $\begin{array}{l}4,8 \\
4,6\end{array}$ & $\begin{array}{l}2,5 \\
1,9\end{array}$ \\
\hline
\end{tabular}


La dernière des deux vitesses étant plus petite, on a reconnu laminaire l'écoulement qui quitte le lit pendant tous nos essais.

Au cours de la fluidisation, la limite de séparation entre la couche fluidisée de flocons et le liquide clair de surface, reste nette jusqu'à la vitesse à vide de $1,5 \mathrm{~mm} / \mathrm{s}$. Sous une vitesse excessive, le fluide commence à entraîner des fragments éparpillés de boue; cet entraînement est dû̀ à la dilution du lit et à l'augmentation du degré de la turbulence. Du point de vue hydrodynamique, il est facile de maintenir la vitesse à vide traversant le lit, même au-dessus de $2,5 \mathrm{~mm} / \mathrm{s}$. Néanmoins, la quantité des matières entrainées par le fluide augsmente, ce qui produit une dégradation de la qualité de l'eau traitée.

$\mathrm{Si}$ on fait diminuer le débit, le niveau du lit descend, ce qui est accompagné d'un accroissement dans la concentration de la boue. La hauteur du lit augmente doucement sous l'afflux d'une suspension fraîche; '’augmentation du lit dépend de la nature de la boue, de la vitesse de traversée du lit et de la qualité de l'eau brute.

Sous une vitesse constante de l'écoulement et sous les différentes hauteurs du lit, les courbes de concentration restent identiques. Il en résulte qu'après la diminution de la vitesse, la concentration de la couche fluidisée de flocons s'épaissit et arrive à la valeur correspondant à la nouvelle vitesse d'écoulement.

Les flocons, faisant partie des suspensions, possèdent deux propriétés principales qui les différencient des particules granuleuses.

1" Une grande surface spécifique, qui fait intervenir la coalescence (tendance des particules floculées à se réunir lorsqu'une certaine concentration est réalisée);

2. Ia fragilité des flocons.

Il y a encore deux autres signes caractéristiques pour le maintien du lit fluidisé de flocons :

$3^{\circ}$ Le lit de boue est composé de particules dont les dimensions sont très différentes;

$4^{\circ}$ Il $\mathrm{y}$ a un changement continu des particules dans le lit: les particules fraîchement floculées s'y écoulent, tandis que les flocons dans le haut du lit sont éloignés par aspiration dans le récipient.

Alors, le mécanisme du lit de bone est influencé par les forces suivantes : le poids des particules dans l'eau, la traînée, les forces turbulentes (c'est-à-dire les forces tangentielles et l'inertie) et les forces de Van der Waals.

Dépendant de Ia hauteur du lit, les rapports, parmi les forces, influent sur la dimension des flocons.
L'analyse hydrodynamique montre où et de quelle facon les impuretés sont écartées de l'eau brute pendant la décantation dans le lit fluidisé des flocons :

Les impuretés floculées sont attirées à la surface des flocons présents au sein de la zone filtrante du lit de boue qui surmonte la zone turbulente. Lorsque la concentration du lit de boue augmente continuellement dans la direction de l'écoulement (de bas en haut), les particules floculées pénètrent jusque dans la couche la plus concentrée. Il en résulte que le traitement de l'eau dans le lit de boue est beaucoup plus avantageux que la filtration dans un lit de sable, dont la porosité est uniforme. Remplaçant le lavage de la couche filtrante, l'aspiration au déversoir élimine la boue excessive, ce qui rend possible l'exploitation continue du décanteur.

La vitesse de l'écoulement étant réduite à $0,7 \mathrm{~mm} / \mathrm{s}$, les flocons les plus lourds descendent vers la toile et la concentration volumétrique de la boue augmente substantiellement. Le fluide cherche une route avec une résistance minimum et commence à former des renards au-dessus de la toile. Par conséquent, la répartition des vitesses devient non uniforme même dans les hauteurs supérieures, ce qui produit la descente et la compression des flocons dans toute la hauteur du lit. Par suite de la différence dans les dimensions des particules, la boue ne se concentre pas uniformément ; sa concentration augmente plus vite dans les couches inférieures du lit. La surface du lit descend, tandis que les renards s'allongent jusqu'à ce qu'ils atteignent toute la hauteur du lit. La figure 9 représente la répartition des concentrations du lit de boue traversé à une vitesse à vide de $0,59 \mathrm{~mm} / \mathrm{s}$ et la descente de son niveau au bout de quelques heures. Lorsque l'eau s'écoule dans les renards, les matières floculées ne sont pas attirées à la surface des flo-

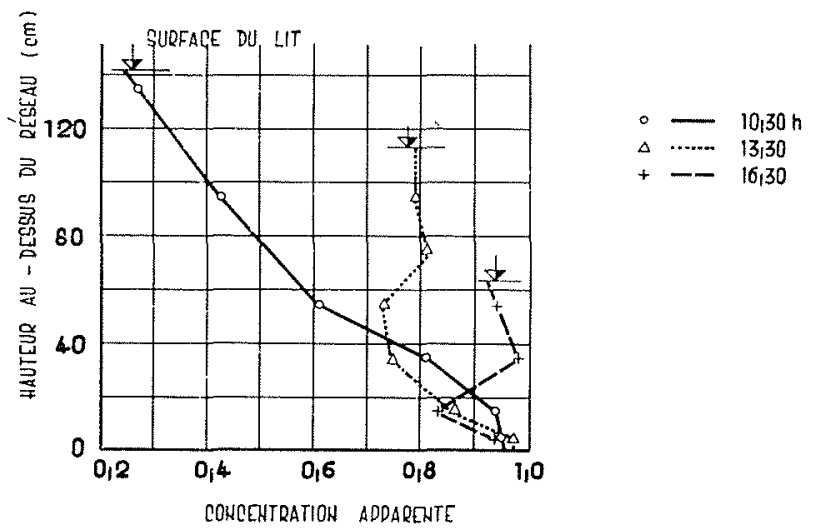

FIG. ?

Répatition des concentrations apparentes de la boue traversée par une vitesse a vide de $0,59 \mathrm{~mm} / \mathrm{s}$, en fonction du lemps. 
cons du lit; elles traversent aussi la boue, produisant une turbidité élevée de l'eau traitée.

Si on arrête l'appareil de dosage, les matières colloïdales cessent de se coaguler pendant la fluidisation particulière; on rompt l'équilibre entre les forces brisant el réunissant les flocons. Dépendant de la force résultante changée, la dimension des flocons décroit. Cependant, les flocons qui deviennent plus légers sont entraînés à une vitesse inchangée du lit; la concentration de la boue s'affaiblit et l'eau entraîne verlicalement les flocons.

Les résultats obtenus dans notre tube expérimental ont démontré que la fluidisation homogène a lieu seulement à des vitesses à vide excédant $0,7 \mathrm{~mm} / \mathrm{s}$.

L'étude présentée a permis d'éclairer les procédés de traitement des eaux ayant lieu dans un type nouveau de décanteur à lit de boue.

\section{REMERCIEMENTS}

Nous tenons ici à exprimer notre gralitude à M. Jean Smetana, membre de l'Académie des Sciences de Tchécoslovaquie, directeur de l'Institut d'Hydrodynamique, pour son appui important et si précieux pour notre travail.
Fn même temps, nous adressons nos remerciements chaleureux à MM. O. Šmek et $\mathrm{Zd}$. Mašek, qui ont bien voulu effectuer les observations exactes et pratiquer les essais.

\section{BIBLIOGRAPHIE}

Jo'trand (R.), Etude de quelques aspects de la fluidisation dans les liquides, Chem. Eng. Sci., 3, pp. 12-16, 1954.

Mackrle (S.), Mackhle (V.), Mřan (V.) : Neue Erkenntnisse über die Filtiation und ihre Anwendung in der Wasserversorgungstechnik, Wasserwirtschaftechnil, sous presse, 1958.

Mackrle (S.), Tesahík (I.), Mackrle (V.), Mičan (V.) : Beobachtungsergebnisse an einer Versuchsanlage zu: Wasseraufbereitung nach dem Flockenwirbelschichtverfahren, Wasservirtschaftechnili, $7, \mathrm{n}^{\circ} 11, \mathrm{pp} .428$ 431,1957 .

Matheson (G, L.), Herbst (W. A.), Holt (P. H.) : Characteristics of fluid-solid systems, Ind. Eng. Chem., 41, No. 6, pp. 1099-1104, 1949.
MoCune (I. K.), Wirmesu (R. H.) : Mass an momentum transfer in solid-liquid system, Ind. Eng. Chem., 41 No. 6, pp. 1124-1134, 1949 .

Monse (R. D.) : Fluidization of granular solids, Ind. Eng. Chem., 41, No. 6, pp. 1117-1124, 1949.

Schaub (F.) : Anwendungen und Grenzen der Wirbelschichttechnik, Chem. Ing. Technik, 24, No. 2, p. 98, 1952 .

TESARík (I.) : Turbulent flow through fluidized beds of identical spheres, Acta Technica (CSR), 2, No. 5 , pp. $410-445,1957$.

WILhelM (R. H.), Kwavk (M) : Fluidization of solid particles, Chem. Eng. Progress, 44, No. 3, pp. 201-218, 1948.

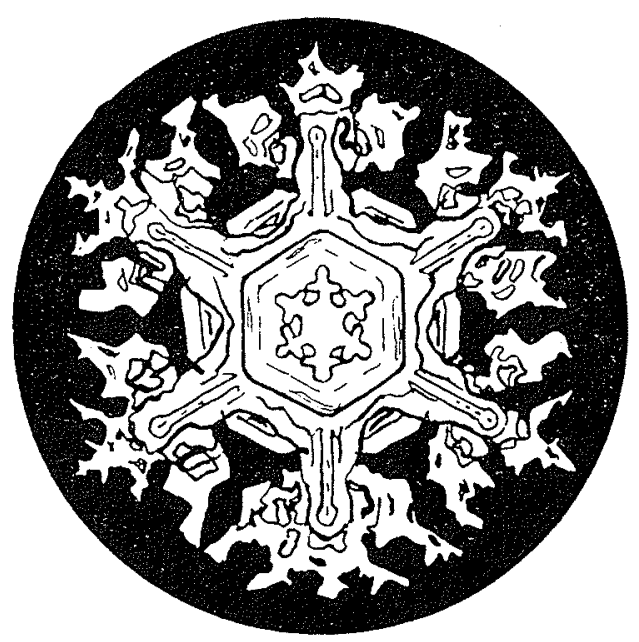

